\title{
Suction Induced Effects on the Fabric of a Structured Soil
}

\author{
AZAD KOLIJI ${ }^{1}$, LYESSE LALOUI ${ }^{1, \star}$, OLIVIER CUSINIER ${ }^{1,2}$ and \\ LAURENT VULLIET ${ }^{1}$ \\ ${ }^{1}$ Ecole Polytechnique Fédérale de Lausanne (EPFL), Laboratoire de mécanique des sols \\ (LMS), CH-1015 Lausanne, Switzerland \\ ${ }^{2}$ Laboratoire Environnement, Géomécanique \& Ouvrages, Ecole Nationale Supérieure de \\ Géologie-INPL, F-54501 Nancy, France
}

(Received: 18 May 2005; accepted in final form: 10 September 2005)

\begin{abstract}
This paper presents the mathematical modelling of the modification of the pore space geometry of a structured soil subjected to suction increase. Structured soil concepts are first introduced considering different fabric units, such as aggregates and fissures. The numerical modelling of the structural evolution is based on experimental test results in which the evolution of the structure of the samples subjected to different suctions is determined using the mercury intrusion porosimetry technique. From this information, the macro and micropore volume evolutions are determined. The results show that drying produces a reduction in the soil total porosity which mainly corresponds to a reduction of the macropore volume. Associated with this phenomenon, an increase in micropore volume is also observed. The proposed model divides pore size distribution into three pore classes (micropores, macropores and non-affected areas). Using the concept of a suction-influenced domain, the proposed model is able to reproduce the main observed fabric evolution between the saturated and dry states.
\end{abstract}

Key words: structured soil, fabric, suction, pore size distribution.

\section{List of notation for mathematical equations}

$R \quad$ The entrance pore radius

$T_{\mathrm{S}} \quad$ Surface tension of the liquid

$\alpha \quad$ Contact angle of fluid interface to solid

$\psi \quad$ Suction

$\psi_{0} \quad$ Initial suction

$\psi_{\mathrm{f}} \quad$ Final suction

$R(\psi) \quad$ The drained pore radius at that value of suction $\psi$

*Author for correspondence: Tel.: +41-21-693-23-14; Fax: +41-21-693-41-53; E-mail: lyesse.laloui@epfl.ch 
$r \quad$ The pore class radius

$v(r, \psi) \quad$ Predicted volume fraction of pore class of $r$ at suction $\psi$

$R_{1}, R_{2}, R_{3} \quad$ Limit radii between pore zones

$V_{\psi} \quad$ Total influenced volume fraction

$V_{\psi \mathrm{e}} \quad$ Effective volume fraction

$V_{3} \quad$ Total volume fraction difference between the initial and final cases in Zone 3

$V_{2} \quad$ Total volume fraction difference between the initial and final cases in Zone 2

$V_{2 \mathrm{e}} \quad$ Total moving volume fraction from macropores to micropores

$C_{2}, C_{3} \quad$ Proportionality coefficients

\section{Introduction}

Natural soils almost always show nested structures, starting from the scale of the grains and pores and their networks up to field scale, with structures that are 5-8 orders of magnitude larger (expressed in their characteristic lengths). Soil structure properties are of great importance in geotechnical, geoenvironmental and agricultural engineering as they influence many soil characteristics, such as compressibility (Lambe, 1958), hydraulic conductivity (Tamari, 1984) or the soil-water characteristic curve (Brustaert, 1968) of both the compacted and natural soils. Consequently, improving the understanding of the structural modifications induced by hydric loads is a key issue. There is a strong relationship between the definition of soil structure and soil heterogeneity. In the first part of this paper, different concepts of structured soils are discussed in order to clarify the approach adopted here.

\subsection{STRUCTURED SOIL}

Natural soils show different kinds of heterogeneity depending on the scale considered. In classical soil mechanics problems, the level of heterogeneity is usually limited to the differences among the characteristics of the soil layers (while at smaller scales, such as that of the pores, heterogeneity can be defined by the means of smaller structural units, such as different pores, fissures or aggregates). Inside a specific soil layer, at a smaller scale, the soil is usually assumed to be homogeneous as soon as enough grains or aggregates are considered, the pore-scale heterogeneities being smoothed out (Laloui et al., 2003). This implies the consideration of a representative elementary volume (REV) of a given material. A REV is required to include a significant amount of small-scale heterogeneity to respect a meaningful statistical average (Vogel and Roth, 1998). As an example, the dependence of the sample porosity on its scale is shown in Figure 1. It can be seen that the REV may be representative of a homogeneous medium, while smaller samples are completely heterogeneous. For volumes below the REV, 


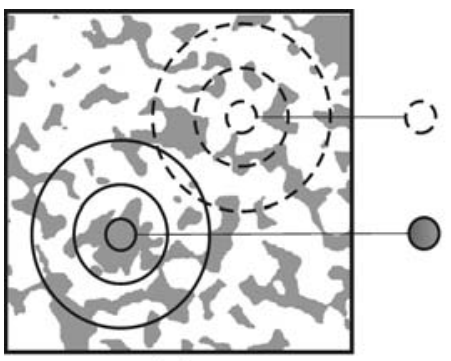

(a)

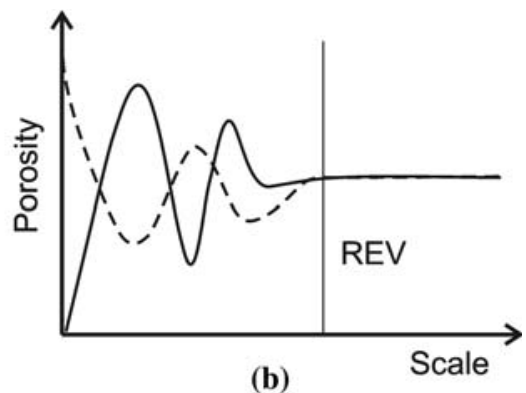

(b)

Figure 1. Effect of sample size on the porosity evolution: (a) Sample sizes; (grey = solid, white $=$ void) (b) Porosity versus sample scale.

as may be seen in Figure 1(a), the whole sample can be located completely within the pores or within the particles, leading to quite different values of porosity. However, when the sample sizes increase, the difference between them decreases and the obtained values of porosity tend to a single one (obtained at the REV level).

Natural soils show different structures, characterised by structural units, such as aggregates, porous block, fissures, earthworm holes and root channels (especially in the top layers of agricultural soils). It is widely accepted in the literature (Griffiths and Joshi, 1990; Gens et al., 1995; Al-Mukhtar et al., 1996; Delage et al., 1996; Cuisinier and Laloui, 2004) that these soils, especially the compacted ones, have two levels of structure: macro and micro. The soil microstructure can be defined as the elementary particle associations within the soil aggregates (Al-Mukhtar et al., 1996), whereas the arrangement of these soil aggregates and the relation among the structural units at the aggregate level is referred to as the macrostructure. Consequently, the pores of a soil consist of two main classes, micropores and macropores, corresponding to the two levels of soil structure. These two different levels play important roles in the hydro-mechanical behaviour and deformation process of soils (Cuisinier and Laloui, 2004). For instance, although micropores are usually neglected, they have a strong influence on water retention characteristics. The larger pores (macropores) dominate the dynamics of solute transport. They also play a crucial role in cases of soil freezing and indirectly affect heat transfer.

\subsection{CONCEPT OF DOUBLE POROSITY}

Such a division of pores into macro and micro levels leads to the concept of double porosity for soils. This concept can be used for soils with two distinct levels of porosity. Figure 2 shows two different types of structured soil for which the concept of double porosity can be used. In 


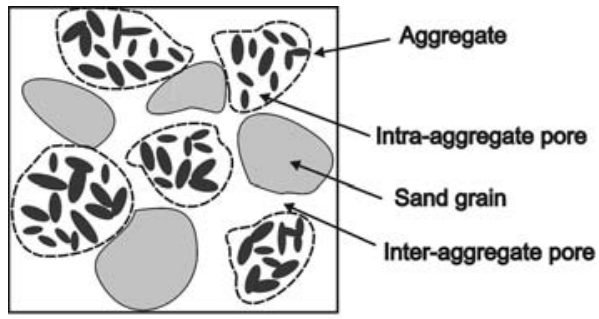

(a)

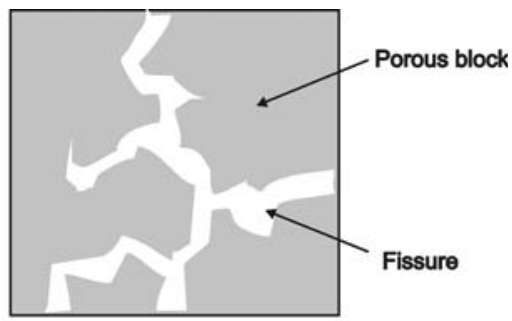

(b)

Figure 2. Structured soils: (a) Aggregated soil; (b) Fissured porous medium.

Figure 2(a), an aggregated soil is presented. Here, the pores between the aggregates (interaggregate) are macropores and the pores within individual aggregates (intraaggregate) form micropores. Figure 2(b) shows a fissured porous medium. It may be thought of as a number of porous blocks separated from each other by a system of randomly distributed fissures. Thus, here again, macropores correspond to fissures and micropores to pores of porous blocks (Valliappan and Khalili, 1990).

Although usually the single porosity models are seen to be fairly successful to describe the behaviour of porous materials, they are not suitable for structured double porosity (fissured or aggregated) materials. In such systems, although most of the fluid mass is stored in the micropores, the permeability of the macropores is much higher than that of the micropores (Tuncay and Corapcioglu, 1995). This leads to a dually permeable medium and also to two distinct fluid pressure fields: one in the macropores and the other in the micropores. Therefore, the fluid pressures contained in the two types of pores may reach equilibrium at different rates, and virtually independently, if the channels for fluid transport between the two types of pores are restricted. This concept leads to models in which distinct pore pressures are present in the matrix and in the fractures (Wang and Berryman, 1996).

The double porosity concept was first introduced by Barrenblatt et al. (1960) as a flow model for non-deformable fissured porous media. This model was simplified by Barrenblatt (1963) and Warren and Root (1963). Then Aifantis (1977) extended this concept by sketching the basis of a multi-porosity model to include mass exchange of solutes through diffusion between the mobile and stagnant water phases. The general theory of Aifantis unified the earlier proposed models of Barenblatt for fluid flow through non-deformable porous media with double porosity and Biot's theory for consolidation of deformable porous media with single porosity. Khalili and Valliappan (1996) added a new coupling link between the elastic volumetric deformations of the two pore systems. Khalili and Selvadurai 
(2003) presented a fully coupled thermo-hydro-mechanical model in elastic media with double porosity.

In most of the previous research work, an attempt was made to explain and to model the hydro-mechanical behaviour of double porous media based on general assumptions concerning soil structure evolution during loading. Very few of them considered unsaturated soils.

\subsection{EXPERIMENTAL CHARACTERISATION OF THE SOIL STRUCTURE}

From the rapid synthesis presented above, soil structure may be seen as the arrangement and organisation of particles in soil. In order to be able to study this soil structure, quantitative approaches are required. One of them is based on the analysis of the pore size distribution (PSD) of soils, widely accepted to be representative of soil structure (Delage and Lefebvre, 1984; Al-Mukhtar, 1995; Delage, et al., 1996). Pore size distribution of soils (normally called soil fabric) corresponds to the geometrical arrangement of soil particles.

In the case of structured double porosity soils, a few studies have been undertaken to characterise fabric changes induced by various types of hydro-mechanical loads. Delage and Lefebvre (1984), studying compacted Champlain clay fabric with mercury intrusion porosimetry (MIP) and scanning electron microscopy, have shown that, during consolidation, only the largest pores collapse at a given stress increment. Small pores are only compressed when all of the macropores have been completely closed by loading. These results were confirmed by other experimental studies (Coulon and Bruand, 1989; Griffiths and Joshi, 1990; Lapierre et al., 1990). Soil fabric is also sensitive to suction increase, as evidenced by other authors (Simms and Yanful, 2001) who investigated the fabric of a compacted glacial till under different suctions, from saturation up to a matric suction of $2500 \mathrm{kPa}$. They have shown that suction increase brought about a progressive rise in the microporosity associated with macroporosity reduction. Simms and Yanful (2001) investigated the relationship between suction and induced fabric modification. Their results have demonstrated that the soil fabric is extremely sensitive to any suction variation. They transformed the measured pore size distribution to take account of pore trapping and developed a model for shrinkage of pores due to suction. Using this model, they proposed a method to predict the changes in pore size distribution during drying tests (Simms and Yanful, 2001, 2002).

In order to model the suction-induced modification in a structured soil, a mathematical model based on experimental results of suction increase on a compacted soil is presented in this paper. The evolution of the double porosity structure of the soil is determined using mercury intrusion porosimetry. The proposed numerical model is presented and validated in Section 3. 


\section{Experimental Results}

As a part of a comprehensive research program, experimental tests were carried out for the analysis of fabric modifications brought about by suction increase (Cuisinier and Laloui, 2004).

The tested soil was a sandy loam from the eastern part of Switzerland (morainic soil of the Swiss central plateau). The plasticity index of the soil was approximately $I_{\mathrm{p}}=12 \%$ and its liquid limit close to $W_{\mathrm{L}}=30 \%$. The unit weight of the soil particles was $\gamma_{\mathrm{s}}=26.1 \mathrm{kN} \mathrm{m}^{-3}$. In this study, all of the samples were prepared using the same procedure. After sampling in the field, the soil was air dried and gently crushed after several days. Aggregates between 0.4 and $2 \mathrm{~mm}$ were selected by sieving and were then wetted up to a mass water content of about $w=14 \%$ and stored in an airtight container for at least 1 week in order to reach moisture equilibrium. The aggregates were then statically compacted directly inside the testing equipment up to a target dry unit density of $\gamma_{\mathrm{d}}=14 \mathrm{kN} \mathrm{m}^{-3}$. In the last stage, the samples were saturated by imposing a low water head, of a few centimetres, at the base of an extra plate apparatus. After this saturation phase, the mass water content of the samples was between 36 and $37 \%$. The initial state of the samples, after the saturation phase, corresponded to zero suction.

In a pressure plate device (air overpressure method for suction control, Richards, 1935), the suction was increased by steps from the saturated state to $50,100,200$ and $400 \mathrm{kPa}$. At each step, when equilibrium was reached, samples were taken for fabric determination by mercury intrusion porosimetry and for water content determination. The obtained water characteristic curve is presented in Figure 3. The repeatability of the procedure was successfully checked and it was shown that the freeze-drying technique used for the MIP had no influence on the pore size distribution (see Cuisinier and Laloui (2004), for more details).

\subsection{SOIL FABRIC DETERMINATION WITH MERCURY INTRUSION POOSIMETRY (MIP)}

Several methods exist to determine soil fabric: scanning electron microscopy, optical microscope, nitrogen adsorption, mercury intrusion porosimetry (MIP), etc. For this study, the MIP technique was selected because it allows the measurement of a wide pore size range, from a few nanometres up to several tens of micrometers, and it permits the identification of the different soil pore classes. The theoretical bases for the determination of soil fabric with MIP are very similar to those of the pressure plate test. In the case of MIP, the non-wetting fluid is mercury and air is the wetting fluid. The mercury pressure is increased by steps and the intruded volume 


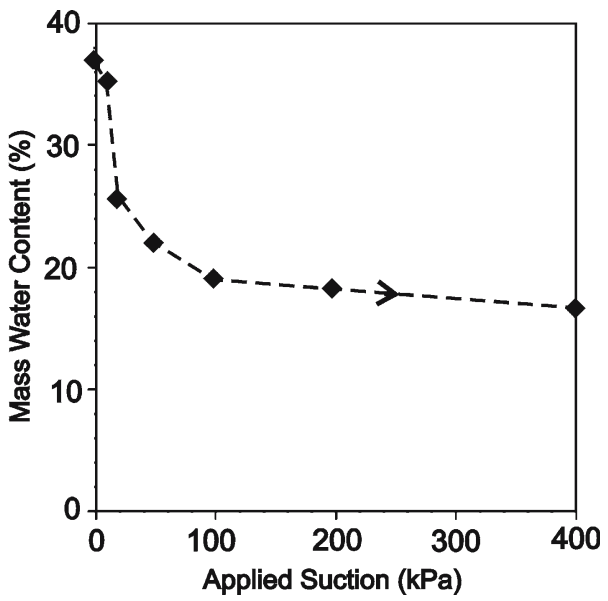

Figure 3. Soil-Water characteristic curve of the tested material.

of mercury is monitored for each pressure increment. Assuming that soil pores are cylindrical flow channels, Jurin's equation is used to determine the pore radius associated with each mercury pressure increment:

$$
R=\frac{2 T_{\mathrm{s}} \cos \alpha}{\psi}
$$

where $R$ is the entrance pore radius, $T_{\mathrm{S}}$ the surface tension of the liquid $\left(0.485 \mathrm{~N} \mathrm{~m}^{-1}\right.$ for mercury), $\alpha$ the contact angle of fluid interface to solid $\left(140^{\circ}\right.$ is an average value for mercury-air interface as suggested in Penumadu and Dean (2000), and $\psi$ the pressure difference between the two interfaces (in Pa). From the MIP test, the injected mercury volume can be measured at each step and the cumulative mercury volume injected can be obtained as a function of the equivalent pore radius. One method to present the results of an MIP test is to give the volume fraction of pores per unit weight of soil sample. This provides physically comparable tools and also facilitates the interpretation of results. Usually due to the wide range of pore radii, i.e. several orders of magnitude, a logarithmic scale is used for the pore radii. It should be pointed out that the determination of PSD with the MIP test is influenced by several experimental side effects, such as pore entrapment, pore neck, etc. As a consequence, the measured PSD might differ from the real PSD of the tested soil and the pore radius associated with each pressure increment is only an entrance pore radius. As a simplification, the term "entrance" will be omitted in the following when dealing with pore radii calculated from MIP data. More details about these phenomena are available in Delage and Lefebvre (1984).

Each soil sample was carefully cut in small pieces of approximately $1 \mathrm{~g}$. Due to technical requirements, the MIP test must be conducted on totally 
dried soil pieces. Among available dehydrating methods, the freeze-drying method was considered as the least disturbing preparation technique for water removal (Gillot, 1973). Soil pieces were quickly frozen with liquid nitrogen (temperature of $-196^{\circ} \mathrm{C}$ ) and then placed in a commercially available freeze-drier for approximately 1 day for the sublimation of the water. The samples were subsequently kept inside desiccators until the MIP tests were performed with a Porosimeter 2000 device (Carlo Erba Instruments).

\subsection{SUCTION INCREASE EFFECT ON PSD}

The pore size distribution (PSD) of the tested material for five suction levels is shown in Figure 4. The PSD of the sample at suction equal to zero (initial state) had a shape very similar to that of a silt sample compacted dry of optimum (Delage et al., 1996), as two pore classes can be seen (micro and macropores). The limit between the two pore classes is about $1 \mu \mathrm{m}$.

Knowing the sample's initial fabric (zero suction), the analysis of the influence of drying on fabric was carried out. It may be seen in Figure 4 that the suction increase induces modifications of the soil fabric, such as a strong reduction of the macroporosity. It is interesting to note that the reduction of the amount of macropores is accompanied by a relative increase in the microporosity, which can be explained by the shrinkage of the macropores. The comparison of all of these PSD curves shows that the drying process did not affect pores smaller than $0.1 \mu \mathrm{m}$.

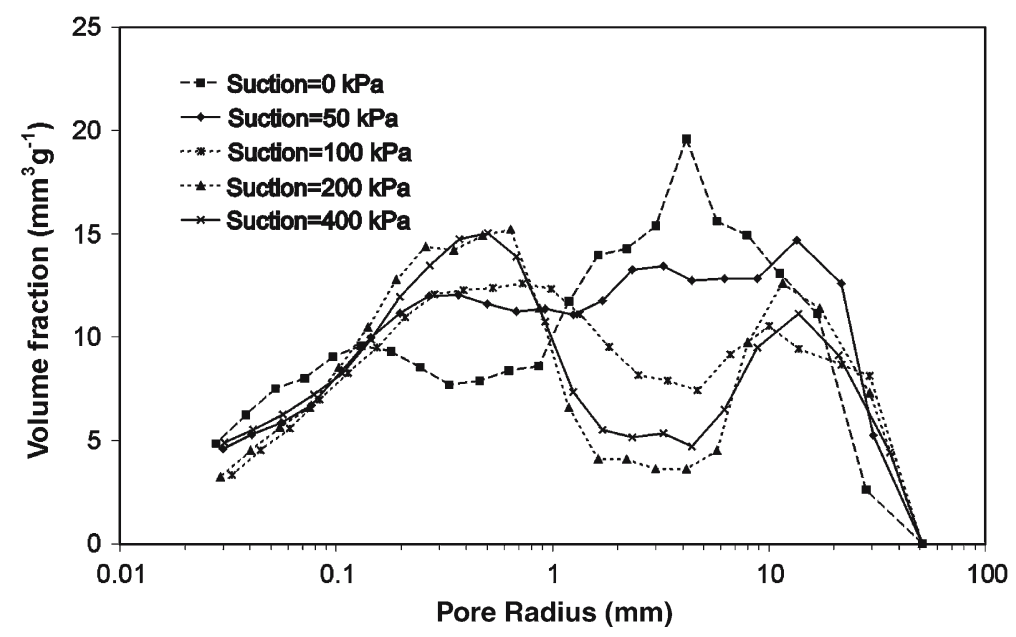

Figure 4. Modification of samples fabric when suction is increased from 0 up to $400 \mathrm{kPa}$. 


\section{Modelling Pore Size Distribution Modification}

In this section, a mathematical model is presented to simulate the PSD modification during the suction increase based on the PSDs of the saturated and the drier cases. Due to the fact that the concept of volume fraction is more suitable for the physical understanding of behaviour, the proposed model was developed on this basis. A validation of the model results with respect to experimental ones then concludes this section.

\subsection{CHARACTERISATION OF PORE CLASSES}

The modelling concept is as follows: giving two limit cases, the saturated case (zero suction - initial case) and the driest case (suction of $400 \mathrm{kPa}$ - final case), we determine the modification of the PSD curves for intermediate cases.

From the results in Figure 5, four different zones of pore classes can be recognised: Zones 1 and 4 are related to the pores which are only slightly affected by suction and on which the influence of suction can be neglected. Zone 2 corresponds to the micropores; the volume fraction of these pores increases as suction increases. The pore volume fraction of the pores that belong to the third zone reduces as suction increases. The behaviour of these pore classes are different and they should be distinguished in the model formulation. Three pore radii $R_{1}, R_{2}$ and $R_{3}$ determine the limits between pore zones.

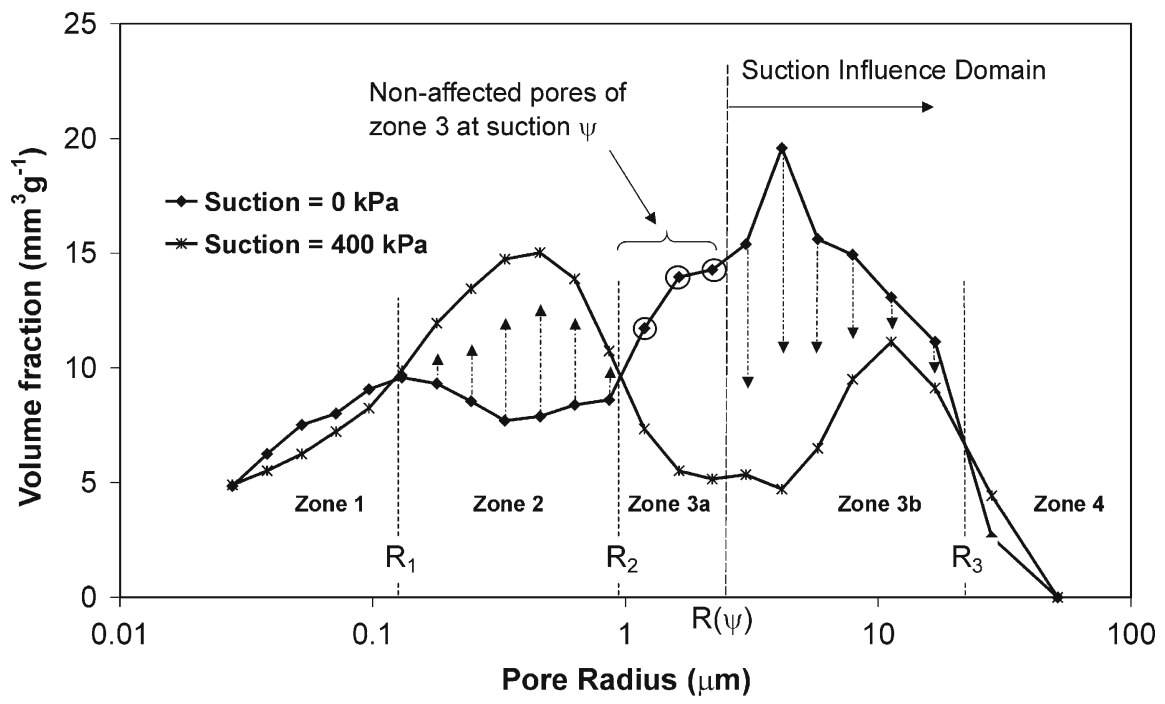

Figure 5. Different zones of pore classes, Suction influence domain and evolution of pore classes. 


\subsection{SUCTION INFLUENCE DOMAIN}

The relationship between any suction $\psi$ and the corresponding pore radius $R$ can be determined by Equation (1) using the value of contact angle $\alpha$ equal to zero (air-water interface):

$$
\psi=\frac{2 T_{\mathrm{s}}}{R(\psi)}
$$

where the surface tension of the water, $T_{\mathrm{s}}$, is equal to $0.07275 \mathrm{~N} \mathrm{~m}^{-1} \cdot R(\psi)$ is the drained pore radius at that value of suction $\psi$ and it can be considered as the minimum pore radius influenced by suction $\psi$ (note that in doing so, hysteretic effects are neglected).

Using Equation (2), it is possible to determine the suction influence domain for a given suction value. This domain is defined as a domain in which the pores have radii greater than $R(\psi)$. Thus, Zone 3 is subdivided into Zones $3 \mathrm{a}$ and $3 \mathrm{~b}$ when $R_{2}<R(\psi)<R_{3}$ (see Figure 5).

\subsection{MODELLING STEPS}

The general framework to model the PSD modifications is outlined as follows:

- volume fraction of pores of Zones 1 and 4 are not affected by suction increase;

- as suction increases, only the pore volume of Zone 3 (macropores) can decrease;

- a part of the volume fraction decrease of Zone 3 will be added to Zone 2 and consequently the volume fraction of Zone 2 (micropores) will increase;

- it is assumed that, at a given suction, only the volume fraction of pores of Zone 3 located in the suction influence domain is added to the volume fraction of pores of Zone 2. This concept is illustrated in Figure 5.

Based on this framework, PSD (or volume fraction distribution) modification at a given suction $\psi$ can be evaluated by considering five intervals of radii.

\subsubsection{Zone 1}

As already mentioned, volume fractions of pores located in Zone 1 are only slightly affected by suction. Therefore, in this model, it is assumed that these pores keep their initial value of volume fraction. This implies:

$$
v(r, \psi)=v\left(r, \psi_{0}\right) ; \quad r<R_{1}
$$


where $r$ is the pore class radius, $v(r, \psi)$ is the predicted volume fraction of pores at suction $\psi$ and $v\left(r, \psi_{0}\right)$ is the volume fractions of pore class measured at the initial stage (zero suction, $\psi_{0}=0$ ).

\subsubsection{Zone 4}

Here again, no changes are expected, and thus:

$$
v(r, \psi)=v\left(r, \psi_{0}\right) ; \quad r>R_{3}
$$

\subsubsection{Zone $3 a$}

For the pores located in Zone 3 but not in the suction influence domain (Zone 3a, Figure 5), no reduction in their volume fraction is experienced and, thus:

$$
v(r, \psi)=v\left(r, \psi_{0}\right) ; \quad R_{2}<r<R(\psi)
$$

\subsubsection{Zone $3 b$}

In this zone, the volume fraction of each pore class can be obtained by subtracting a specific value of volume fraction from the initial PSD. As already assumed for a given suction, only pores of Zone 3 located in the suction influenced domain (Zone $3 b$ ) show reduction in their volume fractions. It can be concluded that the total volume fraction that should be subtracted from the initial case (saturated) during the suction increase from the initial to final case is calculated by:

$$
V_{\psi}=\sum_{r>R(\psi)}\left(v\left(r, \psi_{0}\right)-v\left(r, \psi_{f}\right)\right) ; \quad\left(\text { zone } 3 b, R_{2}<R(\psi)<R_{3}\right)
$$

where $V_{\psi}$ is the total influenced volume fraction, $v\left(r, \psi_{0}\right)$ is the initial volume fraction of pore class with radius $r$ at suction $\psi_{0}$, and $v\left(r, \psi_{\mathrm{f}}\right)$ is the final volume fraction at suction $\psi_{\mathrm{f}}$ of the final case.

It should be noted that in a soil with different pore sizes, the large pores might be entrapped by the smaller ones. It means that some parts of the larger pores are not accessible to be influenced by suction until the smaller pores that traps them are influenced and drained.

As a result of pore trapping, only a portion of $V_{\psi}$ should be subtracted from the initial PSD, called the effective volume fraction, $V_{\psi \mathrm{e}}$. As a first approximation, the relationship between $V_{\psi}$ and $V_{\psi \text { e }}$ can be assumed as:

$$
V_{\psi \mathrm{e}}=C_{3} \cdot V_{\psi}
$$

where $C_{3}=\tilde{C}_{3}(\psi)$ is a function of $\psi$ (to be defined). Knowing $V_{\psi \mathrm{e}}$, the new location of the PSD for a specific suction $\psi$ can be found by linear interpolation between the initial and the final cases: 


$$
v(r, \psi)=v\left(r, \psi_{0}\right)-\frac{V_{\psi \mathrm{e}}}{V_{\psi}} \cdot\left(v\left(r, \psi_{0}\right)-v\left(r, \psi_{\mathrm{f}}\right)\right) ; \quad R(\psi)<r<R_{3}
$$

and by substituting Equation (7) into Equation (8):

$$
v(r, \psi)=v\left(r, \psi_{0}\right)-C_{3} \cdot\left(v\left(r, \psi_{0}\right)-v\left(r, \psi_{\mathrm{f}}\right)\right) ; \quad R(\psi)<r<R_{3}
$$

Due to lack of experimental evidence on pore trapping, an approximation should be made for the value of $C_{3}$. A possible expression for $C_{3}=\tilde{C}_{3}(\psi)$ can be constructed by considering that:

for $R(\psi)<R_{2}, C_{3}=1$ because the PSD curve obtained by MIP is assumed to be the real curve without air trapping.

for $R(\psi)=R_{3}, V_{\psi}=0$ and $C_{3}$ can take any value, $V_{\psi \mathrm{e}}$ being always zero. for intermediate cases, the trapped air is expected to be a function of the ratio $V_{\psi} / V_{3}$, where $V_{3}$ is the total volume fraction difference between the initial and final cases in Zone 3:

$$
V_{3}=\sum_{R_{2}<r<R_{3}}\left(v\left(r, \psi_{0}\right)-v\left(r, \psi_{\mathrm{f}}\right)\right)
$$

Back-calculations from the authors' results (Cuisinier and Laloui, 2004) showed that a possible candidate for $C_{3}=\tilde{C}_{3}(\psi)$ is:

$$
C_{3}=\left(\frac{V_{\psi}}{V_{3}}\right)^{2}
$$

\subsubsection{Zone 2}

In order to model the volume fraction variation of pores in Zone 2, some information about the amount of affected macropore volume fraction which is going to be added to the micropores is required. Volume variation of a soil during the application of suction can be divided in two parts. The first one is the external volume variation which can result in a total porosity reduction, and the second one is the internal volume variation which is related to the shrinkage of macropores and subsequent change into micropores. Therefore, for each macropore class located in Zone 3, there is only a portion of the total experienced volume fraction reduction which is added to the micropore classes. It is assumed that, at each suction step, this portion is determined by a constant coefficient which is equal to the ratio of total micropore increase to the total macropore decrease from the initial to the final case. Therefore, this coefficient can be determined as follows:

$$
C_{2}=\frac{V_{2}}{V_{3}}
$$


where:

$$
V_{2}=\sum_{R_{1}<r<R_{2}}\left(v\left(r, \psi_{\mathrm{f}}\right)-v\left(r, \psi_{0}\right)\right)
$$

The total moving volume fraction from macropores to micropores, $V_{2 \mathrm{e}}$ which is a portion of the total influenced volume fraction $V(\psi)$ is determined by this coefficient:

$$
V_{2 \mathrm{e}}=C_{2} \cdot V_{\psi}
$$

This volume fraction should be distributed among the micropores and added to the initial volume fractions. The new location of the PSD for a specific suction $\psi$ can be found (as in Equation (8)) by linear interpolation. As a result, the modelled volume fraction of pores located in Zone 2 can be estimated by:

$$
v(r, \psi)=v\left(r, \psi_{0}\right)+\frac{V_{2 \mathrm{e}}}{V_{2}} \cdot\left(v\left(r, \psi_{f}\right)-v\left(r, \psi_{0}\right)\right) ; \quad R_{1}<r<R_{2}
$$

\section{Numerical Validation of the Model}

The model presented is conceived for the simulation of the modification of the pore space geometry of double porosity structured soils subjected to a suction increase. It is mainly based on the concept of the suction influence domain and includes experimental evidence of the effect of suction on micropores and macropores. In order to validate this model, it was used to simulate the PSD modification for the experiments presented in Section 2 of this paper.

The initial data needed for the model are the PSD curves for the saturated state (suction of $0 \mathrm{kPa}$ ) and drier state (suction of $400 \mathrm{kPa}$ ). The model predictions made for the PSD curves at suction levels of 50, 100 and $200 \mathrm{kPa}$ will be analysed. The comparison between the simulated curves with the experimental results is given in the following paragraphs.

Table I illustrates the introduced and the obtained data for the intermediate case of suction equal to $50 \mathrm{kPa}$.

The required initial data for modelling are given in the columns 1-3. They consist in radii of the pores that represent different pore classes (column 1), the values of volume fractions at the initial suction $(s=0$, column $2)$ and the final suction $(s=400 \mathrm{kPa}$, column 3$)$. Comparing the initial and the final PSD curves together and considering their intersection at different points, the limits between the zones is determined (Figure 5). Therefore, four different zones are recognised and attributed to the pore classes as it is done in column 4 . In column 5 the pores of zone 3 are divided into zone 


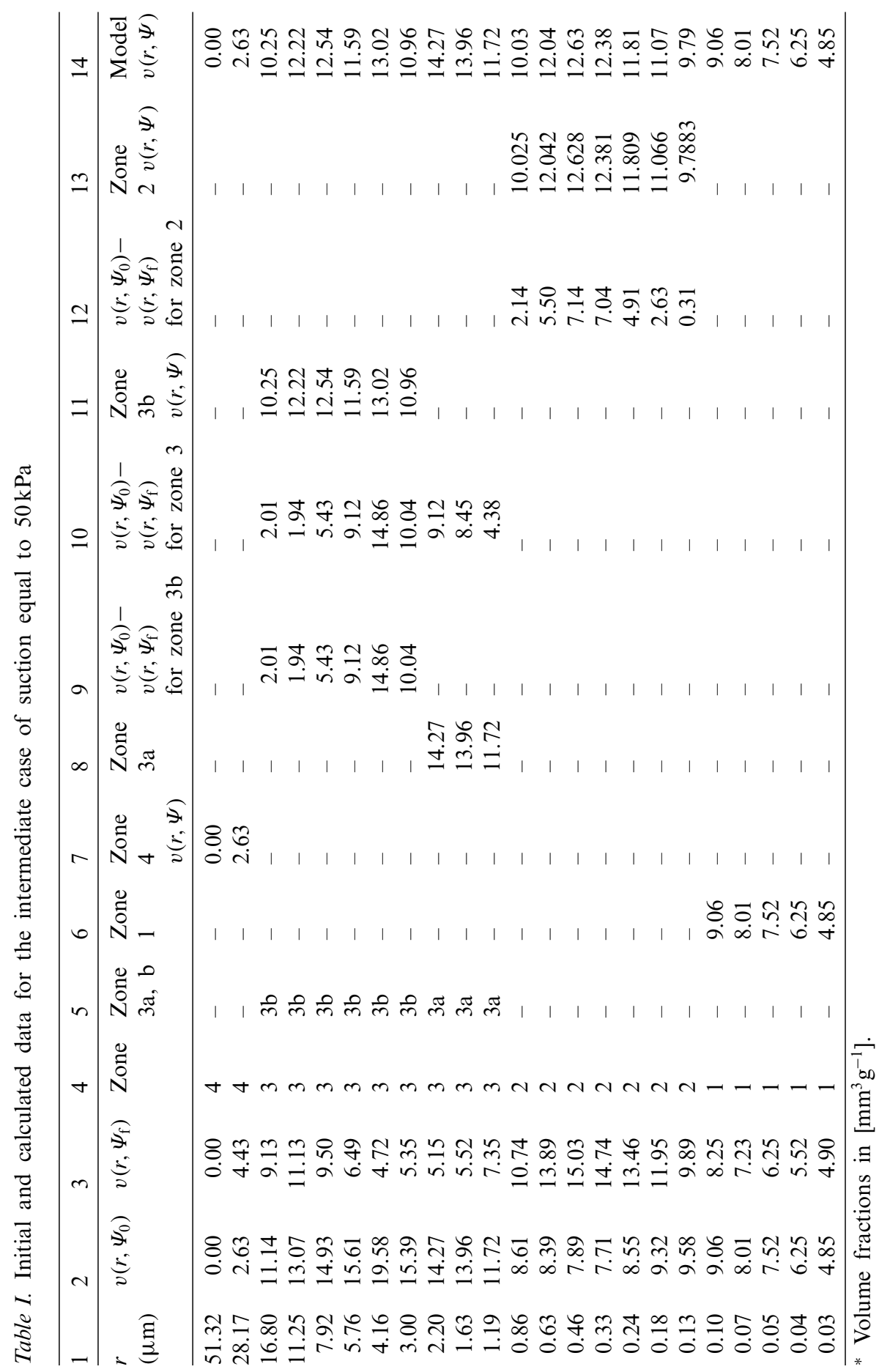


$3 \mathrm{a}$ and $3 \mathrm{~b}$. Zone $3 \mathrm{a}$ represents the pores that have pore radius less than the pore radius corresponding to the current suction $(s=50 \mathrm{kPa})$. According to Equation (1) the corresponding pore radius is equal to $2.91 \mu \mathrm{m}$.

Equations (3)-(5) give the volume fraction of pores at zones 1, 4 and 3a equal to their initial values. This is shown in columns 6,7 and 8 , respectively. To determine the volume fraction of pores in zone $3 \mathrm{~b}$, it is first required to calculate the values of $V_{\psi}$ and $V_{3}$. According to Equations (6) and (10) the values of $V_{\psi}$ and $V_{3}$ are, respectively, equal to the summation of the values of column 9 and 10. In this example, these values are $V_{\psi}=43.40$ and $V_{3}=65.35$ and using these values in Equation (11), one can have $C_{3}=0.441$. Having the value of $C_{3}$ and using Equation (9) the values of volume fraction for zone $3 \mathrm{~b}$ may be calculated. These values are given in column 11 .

$V_{2}, C_{2}$ and $V_{2 \mathrm{e}}$ are the required parameters to calculate the volume fraction of pores in zone 2. $V_{2}$ is calculated using Equation (13), i.e. equal to the summation of column 12. The value of $V_{2}$ here is 29.66. Substituting this value in Equation (12), the value of $C_{2}$ is obtained equal to 0.454. Therefore, according to Equation (14) it is obtained $V_{2 e}=19.70$. Finally, using Equation (15) the values of volume fraction for zone 2 is determined as it is shown in column 13. The model prediction data are then summarised in column 14 .

The same calculation are made for the other intermediate suction values $(s=100,200 \mathrm{kPa})$ and the results are shown in Figure 6. As may be seen in Figure 6(a), the model correctly reproduces the decrease of macropores as well as the increase of micropores. The lower limit domain of the suction influence is situated at a radius of about $3 \mu \mathrm{m}$. The modelled macropores under this limit have the same values of volume fraction as those for the saturated case. This aspect was also observed for the experimental points. The same satisfactory prediction applies to the behaviour at a suction of $100 \mathrm{kPa}$ (Figure 6(b)), even if the experimental and the simulated results are not perfectly superposed. Almost all macropores are affected by the suction increase. The numerical simulation at a suction of $200 \mathrm{kPa}$ is shown in Figure $6(\mathrm{c})$. The model reproduces the behaviour of the three zones remarkably well. In Zones 1 and 4 (pore radius less than $R_{1}$ and greater than $R_{3}$ ) the effect of suction is very limited. In Zone 3 (macropores), the important decrease in the volume fraction is well reproduced. This is also the case for Zone 2 (micropores), where the significant increase in micropores is well predicted. The modelled volume fractions at $200 \mathrm{kPa}$ are the same as those at $400 \mathrm{kPa}$ are due to the fact that this latter one is the final limit of the model.

\section{Conclusions}

In this paper, a mathematical model is presented for the numerical simulation of the modification of the pore space geometry of a structured double 

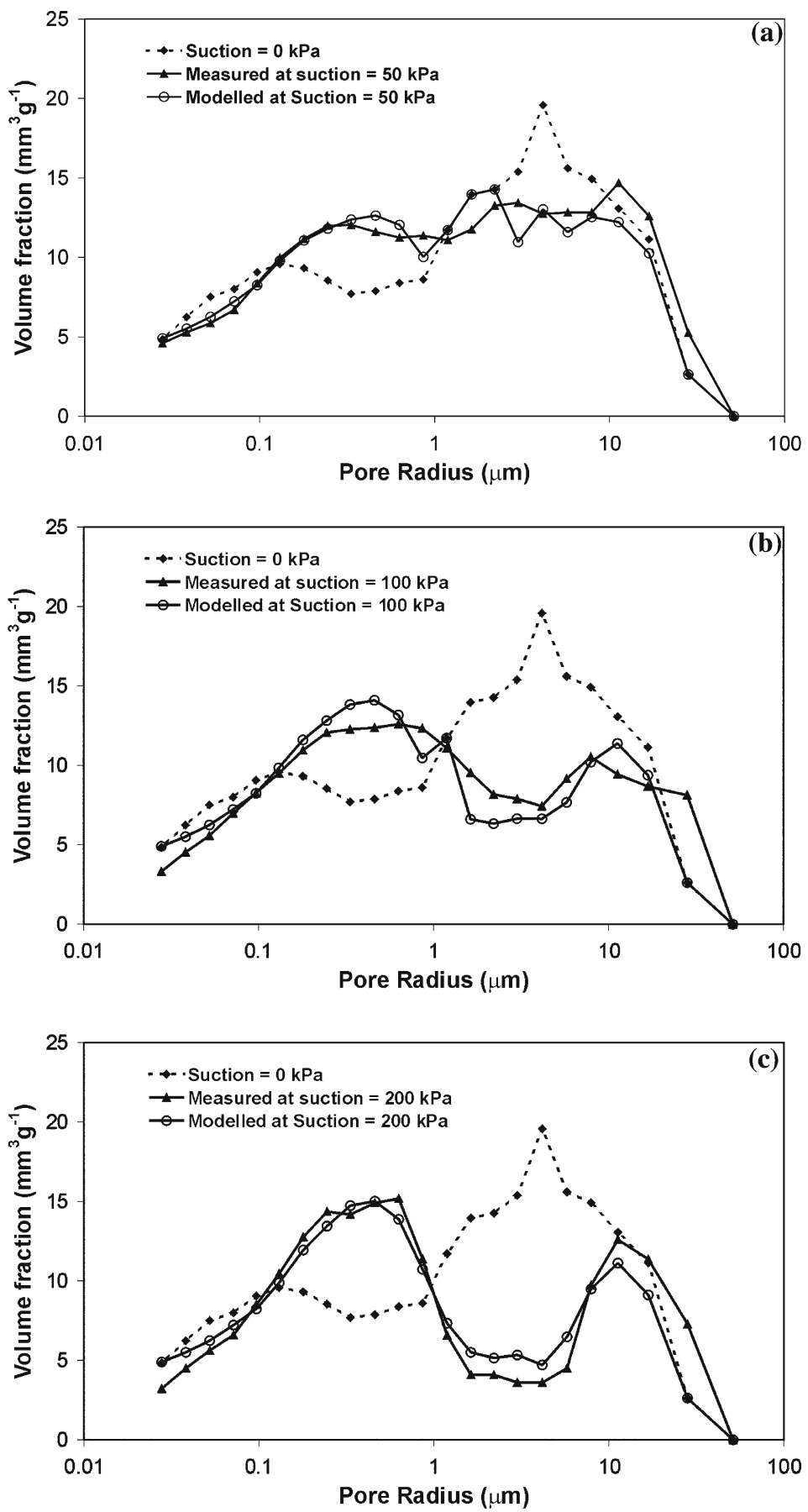

Figure 6. Modelled and measured PSD (a) at suction $50 \mathrm{kPa}$ (b) at suction $100 \mathrm{kPa}$ (c) at suction $200 \mathrm{kPa}$. 
porosity soil subjected to suction increase. The model is mainly based on the concept of the suction influence domain backed up by experimental evidence. The model divides the pore size domain into different zones: macropores, micropores and domains where the suction effect is limited. Each zone has its own behaviour and the micropore and the macropore zones are strongly coupled (a part of the volume lost in the macropores is gained by the micropores). The numerical simulations presented show that the model is able to reproduce the main aspects of suction induced effects on soil structure.

\section{References}

Aifantis, E. C.: 1977, Introducing a multi-porous medium, Dev. Mech. 8, 209-211.

Al-Mukhtar, M.: 1995, Macroscopic behavior and microstructural properties of a kaolinite clay under controlled mechanical and hydraulic state, in: Unsaturated Soils: Proc. 1st Int. Conf. on Unsaturated Soils/UNSAT 95, Paris, pp. 3-9.

Al-Mukhtar, M., Belanteur, N., Tessier, D. and Vanapalli, S. K.: 1996, The fabric of a clay soil under controlled mechanical and hydraulic stress states, Appl. Clay Sci. 11, 99-115.

Barrenblatt, G. I.: 1963, On certain boundary value problems for the equation of seepage of liquid in fissured rock, J. Appl. Math. Mech. (translation of the Soviet journal: Prikladnaja Matematika i Mekhanika (PMM)) 27, 513-518.

Barrenblatt, G. I., Zeltov, I. P. and Kochina, N.: 1960, Basic concepts in the theory of seepage of homogeneous liquids in fissured rocks, J. Appl. Math. Mech. (translation of the Soviet journal: Prikladnaja Matematika i Mekhanika (PMM)) 24(5), 1286-1303.

Brustaert, W.: 1968, The permeability of a porous medium determined from certain probability laws for pore-size distribution, Water Resour. Res. 4, 425-434.

Coulon, E. and Bruand, A.: 1989, Effects of compaction on the pore space geometry in sandy soils, Soil Till. Res. 15, 137-152.

Cuisinier, O. and Laloui, L.: 2004, Fabric evolution during hydromecanical loading of a compacted silt, Int. J. Numer. Anal. Meth. Geomech. 28(6), 483-499.

Delage, P., Audiguier, M., Cui, Y.-J. and Howat, M.D.: 1996, Microstructure of compacted silt, Can. Geotech. J. 33, 150-158.

Delage, P. and Lefebvre, G.: 1984, Study of the structure of a sensitive champlain clay and of its evolution during consolidation, Can. Geotech. J. 21(1), 21-35.

Gens, A., Alonso, E. E., Suriol, J. and Lloret, A.: 1995, Effect of structure on the volumetric behavior of a compacted soil, in: Unsaturated Soils: Proc. 1st Int. Conf. on Unsaturated Soils/UNSAT 95, Paris, pp. 83-88.

Gillot, J. E.: 1973, Methods of sample preparation for microstructural analysis of soil, in: Soil Macroscopy - 4th International Working-Meeting on Soil Micromorphology, Jamaica, Kingston, pp. 143-164.

Griffiths, F. j. and Joshi, R. C.: 1990, Change in pore size distribution due to consolidation of clays, Geotechnique 40(2), 303-309.

Khalili, N. and Selvadurai, A. P. S.: 2003, A fully coupled constitutive model for thermo-hydro-mechanical analysis in elastic media with double porosity, Geophys. Res. Lett. 30(24), SDE 7-1-SDE 7-5.

Khalili, N. and Valliappan, S.: 1996, Unified theory of flow and deformation in double porous media, Eur. J. Mech. A-Solid 15(2), 321-336. 
Lambe, T. W.: 1958, The engineering behaviour of compacted clays, J. Soil Mech. Found. Div. ASCE 84, 1-35.

Laloui, L., Klubertanz, G. and Vulliet, L.: 2003, Solid-Liqiud-Air coupling in multiphase porous media, Int. J. Numer. Anal. Meth. Geomech. 27, 183-206.

Lapierre, C., Leroueil, S. and Locat, J.: 1990, Mercury intrusion and permeability of Louisville clay, Can. Geotech. J. 27, 761-773.

Penumadu, D. and Dean, J.: 2000, Compressibility effect in evaluating the pore-size distribution of Kaolin clay using mercury intrusion porosimetry, Can. Geotech. J. 37(2), 393-405.

Richards, R. A.: 1935, Capillary conduction of liquids through porous medium, Physics 1, $318-333$.

Simms, P. H. and Yanful, E. K.: 2001, Measurement and estimation of pore shrinkage and pore distribution in a clayey till during soil-water characteristic curve tests, Can. Geotech. J. 38(4), 741-754.

Simms, P. H. and Yanful, E. K.: 2002, Predicting soil-water characteristic curves of compacted plastic soils from measured pore-size distribution, Geotechnique 52(4), 269-278.

Tamari, S.: 1984, Relation between pore-space and hydraulic properties in compacted beds of silty-loam aggregates. Soil Technol. 7, 57-73.

Tuncay, K. and Corapcioglu, M. Y.: 1995, Effective stress principle for saturated fractured porous media, Water Resour. Res. 31(12), 3103-3106.

Valliappan, S. and Khalili, N.: 1990, Flow through fissured porous media with deformable matrix, Int. J. Neumer. Meth. Eng. 29, 1079-1094.

Vogel, H. J. and Roth, K.: 1998, A new approach for determining effective soil hydraulic functions, Eur. J. Soil Sci. 49, 547-556.

Wang, H. F. and Berryman, J. G.: 1996, On constitutive equations and effective stress principles for deformable, double-porosity media, Water Resour. Res. 32(12), 3621-3622.

Warren, J. R. and Root, P. J.: 1963, The behaviour of naturally fractured reservoirs, Soc. of Petrol. Eng. J. 245-255. 